\title{
Acute Quadriceps Compartment Syndrome and Rhabdomyolysis in a Weight Lifter Using High-Dose Creatine Supplementation
}

\author{
Stacey J. Robinson, CAPT, USAF MC
}

Creatine, a nutritional supplement marketed to increase strength and muscle mass, is the most profitable performance-enhancing agent used among athletes today, grossing more than $\$ 200$ million in 1998. ${ }^{1}$ As its use increases, especially among highschool athletes, the concern about its safety mounts. Because creatine is a dietary supplement, it is not regulated by the Food and Drug Administration, and distributors are not required to give any specific dosing recommendations.

Researchers recommend a loading dose of 20 to $25 \mathrm{~g} / \mathrm{d}$ for 5 to 7 days followed by a maintenance dose of 2 to $5 \mathrm{~g} / \mathrm{d}$ for oral supplementation. ${ }^{2} \mathrm{Al}-$ though studies investigating the benefits of creatine report no evidence of side effects, most of these studies observed patients only during the loading period. A few studies observed patients during the maintenance period, but the longest study lasted only 10 weeks. ${ }^{3}$ There are no studies investigating the side effects of creatine supplementation beyond 10 weeks or with higher maintenance doses. Many distributors and sources in the popular media advocate a higher dose than recommended. Furthermore, a survey by Juhn and colleagues ${ }^{4}$ found that 39 of 52 athletes who supplemented their diets with creatine exceeded the recommended maintenance dose. The following case of a weight lifter who developed acute compartment syndrome raises suspicion regarding the safety of high-dose, long-term creatine use.

\section{Case Report}

A 24-year-old previously healthy man came to the emergency department complaining of severe bi-

Submitted, revised, 18 August 1999.

From the Department of Family Practice, 6th Medical Group, MacDill Air Force Base, Fla. Address reprint requests to Stacey J. Robinson, MD, Department of Family Practice/SGOPF, 8415 Bayshore Blvd, MacDill AFB, FL 33621. lateral thigh pain on awakening, which gradually worsened during the day to the point that he could no longer walk. On the day before his visit, the patient participated in approximately 3 hours of lower extremity exercise. For the preceding 8 days, he had not exercised because of a minor muscle strain. The patient had been an avid body builder for approximately 5 years and reported that he had performed similar lower extremity exercise without any problems. For the past year he had been using oral creatine supplementation at five times the recommended maintenance dose $(25 \mathrm{~g} / \mathrm{d})$. The remainder of his medical history was unremarkable except for occasional alcohol use (average of 4 drinks per month). The patient denied use of tobacco, anabolic steroids, illicit drugs, or other ergogenic supplements. Review of systems was only notable for onset of gross hematuria shortly after his arrival at the emergency department.

In the emergency department, his blood pressure, respirations, temperature, and heart rate were normal. Findings on his physical examination were normal except for bilaterally tense, edematous anterior thighs and severe pain with any degree of knee flexion. Initial laboratory studies were serum sodium $149 \mathrm{mEq} / \mathrm{L}$, potassium $4.2 \mathrm{mEq} / \mathrm{L}$, blood urea nitrogen $22 \mathrm{mg} / \mathrm{dL}$, creatinine $1.0 \mathrm{mg} / \mathrm{dL}$, creatine phosphokinase $131,000 \mathrm{U} / \mathrm{L}$, and a calculated serum osmolality of $311 \mathrm{mOsm} / \mathrm{L}$. Urinalysis showed proteinuria $(3+)$, hematuria $(4+), 10$ to 15 red blood cells per high-power field and 80 to 100 red cell casts per high-power field. Of note, serum calcium and phosphorus and urine myoglobin were not measured. The patient had no previous laboratory reports on file for comparison. Acute compartment syndrome was suspected and confirmed by anterior, medial, and lateral quadriceps compartment pressures of 34,34 , and $32 \mathrm{~mm} \mathrm{Hg}$ on the right and 17,15 , and $15 \mathrm{~mm} \mathrm{Hg}$ on the left, respectively. The patient was admitted for treatment 
of acute quadriceps compartment syndrome and rhabdomyolysis and underwent emergency bilateral tri-compartment fasciotomies. Left fasciotomies were done despite normal compartment pressures because the patient had severe symptoms on the left. Perioperatively, the rhabdomyolysis was treated with aggressive hydration with crystalloid and urine alkalinization and then diuresis.

During the first several days of hospitalization, the patient's creatinine levels began to rise, and he developed edema of the lower extremities extending up to the flanks. On hospital day 6 , he developed increasing dyspnea and hypoxia. The patient developed a new grade $2 / 6$ systolic murmur at the left upper sternal border. A chest radiograph showed cardiomegaly, pulmonary venous congestion, and bilateral effusions. An electrocardiogram showed a left axis deviation of $-38^{\circ}$ and incomplete right bundle branch block. An echocardiogram showed an ejection fraction of $48 \%$, concentric left ventricular hypertrophy, and mild mitral and tricuspid regurgitation. The pulmonary edema was believed to be secondary to iatrogenic fluid overload, although it was unclear whether the echocardiogram findings were preexisting or temporarily associated with the acute illness. During the remainder of the hospital course, his pulmonary edema gradually resolved with aggressive diuresis. The rhabdomyolysis and acute renal failure resolved with hydration and urine alkalinization with a maximum creatine kinase of $>800,000 \mathrm{U} / \mathrm{L}$ and creatinine of $3.1 \mathrm{mg} / \mathrm{dL}$. The patient required aggressive inpatient physical therapy until he was able to walk independently. After a 22-day hospital course, he was discharged for continued outpatient physical therapy.

At a 6-month follow-up visit, the patient had returned to work but was still undergoing aggressive physical therapy and had achieved approximately $60 \%$ of his premorbid quadriceps strength. At that time an echocardiogram showed high-normal left ventricular thickness, ejection fraction of $55 \%$, and mild right ventricular enlargement without further evidence of mitral or tricuspid regurgitation. A follow-up echocardiogram at 12 months again showed mild increased left ventricular thickness, which was within normal limits when calculated with respect to body surface area. The patient is no longer taking creatine or any other performance-enhancing agents because he believes that use of creatine led to this condition.

\section{Discussion}

Creatine functions as a high-energy phosphate carrier, donating a phosphate for the recycling of adenosine triphosphate (ATP) from adenosine diphosphate (ADP), providing energy for muscle contraction. The daily requirement of creatine for a $70-\mathrm{kg}$ person is $2 \mathrm{~g}$. Creatine is obtained from dietary consumption of meat and fish as well as synthesis from endogenous amino acids. There is approximately $1 \mathrm{~g}$ of creatine contained in 8 ounces of lean beef.

In theory, oral supplementation with creatine will increase muscle creatine stores, providing higher rates of ATP recycling and better performance. In 1992 Harris et $\mathrm{al}^{5}$ were the first to show an increase $(20 \%)$ in total muscle creatine after an oral creatine load (20 to $25 \mathrm{~g}$ for 5 to 7 days). Results of numerous studies investigating the performance-enhancing effects of creatine have not been as consistent. In a 1998 review of the performance-enhancing effects of creatine, Juhn and Tarnopolsky ${ }^{6}$ reported that creatine has consistently been shown to improve only repeated 6- to 30second bouts of stationary cycling with recovery of 20 seconds to 5 minutes. Effects of creatine on single cycling sprints and single or repeated swimming and running sprints have been too inconsistent to draw any conclusion. They also report that creatine does not benefit submaximal or endurance exercise. Of note, most studies were performed in a laboratory setting; therefore, effects on actual competition are unknown.

Hultman et $\mathrm{al}^{2}$ also state that lean body mass is increased by creatine supplementation. Although the mechanism is unclear, it is believed that shortterm gains in body mass are secondary to fluid retention, because creatine draws free water into the muscle cells. This cellular hydration could stimulate protein synthesis, leading to a long-term increase in true muscle mass. ${ }^{7}$

Doubts about the safety of creatine were first raised when three college wrestlers, who were reportedly taking creatine, died within a 2 -month period in $1997 .^{8}$ Dehydration was evident in all three cases, and causes of death were documented as unknown, hyperthermia, and rhabdomyolysis, respectively. They had all been participating in rapid weight-loss techniques, including intensive exercise in rubber suits and saunas, in an attempt to qualify for a lower weight class. Although wrestlers 
have been using rapid weight-loss techniques for decades, these are the first documented cases of deaths in collegiate wrestling. Although the Centers for Disease Control and Prevention and the FDA has not implicated creatine in the wrestlers' deaths, it is difficult to ignore that creatine was a new factor common to these wrestlers and might have been the additional contributor leading to fatal effects of severe dehydration.

Anecdotal reports of side effects include gastrointestinal symptoms, muscle cramps and strains, dehydration, renal and liver dysfunction, and seizures. None of these potential side effects has been substantiated, although studies have involved relatively few subjects. A review of the literature found only two cases involving adverse effects of creatine use. A 25-year-old male soccer player with a history of focal segmental glomerulosclerosis, who had maintained normal renal function on cyclosporin, had serious worsening of renal function after taking a creatine load of $15 \mathrm{~g} / \mathrm{d}$ for 1 week then $2 \mathrm{~g} / \mathrm{d}$ for 6 weeks. Renal function normalized when the creatine was discontinued. 9 The second case report described a previously healthy 20 -year-old man who, after taking $20 \mathrm{~g}$ of creatine daily for 4 weeks, developed interstitial nephritis that also resolved when creatine was discontinued. ${ }^{10}$ Two in vitro studies from the 1970s might link creatine use with cardiac muscle hypertrophy. ${ }^{11,12}$

As do many other athletes, this patient was taking long-term, high-dose creatine. He developed acute compartment syndrome after a lower extremity workout similar to his previous workouts. Based on what is known about its effects on the distribution of total body water, creatine could have predisposed this patient to compartment syndrome by increasing water content in the muscle cells and thus increasing baseline compartment pressures. Another contributing factor could have been the 8-day rest period preceding the event, which brings into question the safety of taking a loading dose of creatine in the absence of exercise. Furthermore, creatine could have predisposed this patient to dehydration, contributing to the development of acute renal failure by causing intravascular volume depletion. Without a baseline echocardiogram for comparison, it is unclear whether creatine contributed to left ventricular hypertrophy and cardiac decompensation.

\section{Conclusion}

Creatine is the most profitable nutritional supplement marketed today, used by professional, college, recreational, and even high-school athletes. Physicians should inquire about creatine use and be prepared to discuss its potential risks and benefits. Because creatine is not FDA-regulated, manufacturers are not required to give any dosing recommendations, and there is no safety profile. Furthermore, the risks of higher doses and long-term use are unknown. As for many other nutritional supplements, studies investigating the side effects of creatine are lacking, and patients must consider the risks of taking a non-FDA-regulated supplement. Although a causal relation cannot be proved with respect to creatine and the patient described in this article, this case raises further suspicion regarding the safety of creatine with higher doses and longterm use.

\section{References}

1. Schnirring L. Creatine supplements face scrutiny. The Physician and Sportsmedicine 1998;26(6):1523.

2. Hultman E, Soderlund K, Timmons JA, Cederblad G, Greenhaff PL. Muscle creatine loading in men. J Appl Physiol 1996;81:232-7.

3. Vandenberghe K, Goris $M$, Van-Hecke P, Van-Leemputte M, Vangerven L, Hespel P. Long-term creatine intake is beneficial to muscle performance during resistance training. J Appl Physiol 1997;83:205563.

4. Juhn MS, O'Kane JW, Vinci DM. Oral creatine supplementation in male collegiate athletes: a survey of dosing habits and side effects. J Am Diet Assoc 1999;99:593-5.

5. Harris RC, Soderland K, Hultman E. Elevation of creatine in resting and exercised muscle of normal subjects by creatine supplementation. Clin Sci (Colch) 1992;83:367-74.

6. Juhn MS, Tarnopolsky M. Oral creatine supplementation and athletic performance: a critical review. Clin J Sport Med 1998;8:286-97.

7. Haussinger D, Roth E, Lang F, Gerok W. Cellular hydration state: an important determinant of protein catabolism in health and disease. Lancet 1993;341: 1330-2.

8. Hyperthermia and dehydration-related deaths associated with intentional rapid weight loss in three collegiate wrestlers-North Carolina, Wisconsin, and Michigan, November-December 1997. MMWR Morb Mortal Wkly Rep 1998;47:105-8.

9. Pritchard NR, Kalra PA. Renal dysfunction accompanying oral creatine supplements. Lancet 1998;351: 1252-3. 
10. Koshy KM, Griswold E, Schneeberger EE. Interstitial nephritis in a patient taking creatine. $N$ Engl J Med 1999;340:814-5.

11. Ingwall JS, Morales MF, Stockdale FE, Wildenthal $\mathrm{K}$. Creatine: a possible stimulus for skeletal and car- diac muscle hypertrophy. Recent Adv Stud Cardiac Struct Metab 1975;8:467-81.

12. Ingwall JS. Creatine and the control of muscle-specific protein synthesis in cardiac and skeletal muscle. Circ Res 1976;38(5 Suppl 1):1115-23. 